\title{
Checklist procedures and the cost of automaticity
}

\author{
IMMANUEL BARSHI and ALICE F. HEALY \\ University of Colorado, Boulder, Colorado
}

\begin{abstract}
Automaticity is usually discussed in terms of its benefits. Automaticity has, however, a cost that manifests itself in procedures that are highly routinized but require close attention, such as verbal checklist procedures. In such procedures, errors occur because the routine leads to automaticity. In three paper-and-pen experiments, we tested this manifestation and investigated ways to decrease automaticity in verbal checklist procedures. In the experiments, subjects proofread sets of multiplication problems to detect erroneous operations, simulating the checklist procedure. In Experiments 1 and 2, two conditions were compared: a fixed-order condition (in which each set contained operations in the same order) and a varied-order condition (in which the operations were in a different order in each set). In Experiment 1, proofreading times were measured to establish the role of fixed sequential order as a consistent environment promoting the emergence of automaticity. In Experiment 2, we introduced errors into the material, and in Experiment 3 we introduced "alerting" conditions to interfere with the development of automaticity. The results indicated that the subjects in the varied-order and alert conditions detected significantly more errors than did those in the fixed-order condition. The implications of the findings for current theories of automaticity are discussed as well as those for the design of checklist procedures.
\end{abstract}

Verbal checklist procedures constitute crucial parts of complex operations, such as flying aircraft and operating nuclear power plants (see, e.g., Degani \& Wiener, 1990; Swain \& Guttman, 1983, for further discussion of checklist applications). In modern aviation, each phase of flight is conducted according to a checklist. In most airline operations, performing the checklist follows the challenge-response procedure. In this procedure, the pilot not flying (PNF) reads the items to be checked from a printed list. Often, the pilot flying (PF) performs the called-for check, verifies the proper execution, and responds verbally to the challenge. Usually, this response includes the called item with the addition of the word "set" or "checked." In some cases, the response includes the specific indication of the checked item (e.g., gauge reading, control-position indicator reading), whereas in other cases, the response may be just "checked."

The routinization (or "ritualization') of checklist procedures was clearly illustrated on March 23, 1983, when the crew of a Frontier Airline Boeing 737 inbound to Casper, Wyoming, went through their routine prelanding checklist. The Captain's (PNF) call "gear down" (referring to the landing gear) was properly responded to by the Copilot's (PF) "gear down three greens" (referring to the three green lights that indicate that the nose wheel and the main landing gear under the wings are down

\footnotetext{
This research was supported in part by Army Research Institute Contract MDA $903-90-\mathrm{K}-0066$ to the Institute of Cognitive Science at the University of Colorado. We are indebted to Lyle Bourne and Anders Ericsson for valuable discussions about this research, to Bill Marmie and Tim Rickard for help with the computer, to Ursula Williams for help with testing the subjects in Experiment 3, and to Susan Dumais, Gordon Logan, and two anonymous reviewers for their helpful comments on earlier versions of this article. Correspondence should be addressed to I. Barshi, Department of Linguistics, Campus Box 295, University of Colorado, Boulder, CO 80309-0295.
}

and locked in their down position). The subsequent gearup landing (landing on the airplane's belly instead of the extended landing gear) reminded the crew and the rest of the aviation community that the countless repetition of the same procedure can lead to a dangerous automatization.

Following the relevant checklist is an integral part of every phase of flight. However, going through the same identical procedure time after time without any variation might lead to automaticity. The mistake made by the Frontier crew is not uncommon and is, therefore, important to understand. The following preliminary study is a first step in the investigation of checklist ritualization.

The term communication ritual refers to situations in which the exchange of phrases is fixed by social conventions. In such exchanges, the literal meaning of the words used is of little relevance to the social context. Greetings, as in the exchange "How ya doin?" "Fine. How about you?" make for a well-established set of rituals. In such rituals, the literal meaning of the message may be ignored, although the participation in the exchange in terms of supplying the proper response is obligatory, and failure to do so constitutes a violation of the social code. Such a violation is likely to raise questions by the initiator, who may even be offended (see, e.g., Clark, 1979; Gumperz \& Hymes, 1972; Schieffelin \& Ochs, 1986, for further discussion of communication rituals). This phenomenon of going through the motions without paying attention to some of the details has also been studied by social psychologists under such labels as "mindlessness"' (see, e.g., Bargh, 1984; Langer, 1989).

The checklist procedure in the cockpit can also become a ritual in which the initiated call must be answered with the proper response while the actual meaning and required action may be dissociated from the verbal exchange and, thus, ignored. As with the communication ritual, failure to supply the expected verbal response constitutes a vio- 
lation. Such failure will be easily detected by the initiating pilot and will trigger a question or a re-call of the item to be checked. The proper response, being the obligatory part of the exchange, will not trigger any questions and the PNF is most likely to continue with the next item on the list. In cases in which the crew members know each other well and trust each other's professionalism, or under time pressures and distractions, the PNF is especially unlikely to check the PF's proper execution of the checked item, relying on the verbal response only (Degani \& Wiener, 1990; Hawkins, 1987). This reliance on the verbal exchange can easily lead to hazardous situations, as in the Frontier Airline example.

Although communication rituals are a harmless natural part of everyday life, the ritualization of checklist procedures on the flight deck harbors danger. To prevent this danger, the ritualization process must be interrupted and stopped. To identify and control the process, it must be thoroughly understood. In this study, we propose to understand the ritualization process in terms of automatic processing, and its prevention in terms of controlled processing. By so doing, we are extending the application of the concepts of automatic and controlled processing beyond the traditional realm under which they have previously been examined.

An important development in refining the notions of controlled and automatic processing has been the work of Schneider and Shiffrin (Schneider \& Shiffrin, 1977; Shiffrin \& Schneider, 1977). Automatic processing became the explanation for fast, effortless performance resulting from consistent training, whereas controlled processing explained slow, effortful performance (for review, see Schneider, Dumais, \& Shiffrin, 1984). Since the publication of the original work by Schneider and Shiffrin in 1977 , automaticity has been the source of explanation for a variety of experimental data, and of application to training in particular (see, e.g., Fisk, Ackerman, \& Schneider, 1987; Fisk \& Eboch, 1989; Fisk \& Gallini, 1989; Logan, 1988a, for further discussion of training applications). Automaticity has also been the center of much controversy (see, e.g., Cheng, 1985; Duncan, 1986; Logan \& Klapp, 1991; Ryan, 1983). Logan and Klapp (1991) consider that controversy to be a problem "with the method of defining automaticity and not with the concept of automaticity itself' (p. 191). However, even if a definition of automaticity is universally accepted, explaining automaticity itself remains a problem.

Currently, the common theoretical approaches to automaticity include property-list approaches, resource theory, and automaticity as memory. Logan and Klapp (1991), who advocate the automaticity-as-memory approach, offer the following critique of the other two approaches:

Approaches that define automaticity in terms of manifest properties, such as speed, effortlessness, and autonomy (property-list approaches), are stipulative or descriptive but not predictive (e.g., Hasher \& Zacks, 1979; LaBerge \& Samuels, 1974; Shiffrin \& Schneider, 1977). They provide no underlying mechanism from which predictions about the properties of automaticity ... can be deduced. Approaches that define automaticity in terms of resource theory specify an underlying mechanism, arguing that automatic processing is (relatively) resource free (e.g., Logan, 1978; Posner \& Snyder, 1975). The resource mechanism allows properties of automaticity to be deduced .... However, resource the ories do not specify a learning mechanism. (p. 179)

In automaticity-as-memory theory, "performance is automatic when it is based on direct-access, single-step retrieval of solutions from memory rather than some algorithmic computation (Logan, 1988b; Newell \& Rosenbloom, 1981; Rosenbloom \& Newell, 1986; Schneider, 1985; Schneider \& Detweiler, 1987)" (Logan \& Klapp, 1991, p. 179). According to Logan and his colleagues (Klapp, Boches, Trabert, \& Logan, 1991; Logan, 1988b; Logan \& Klapp, 1991), automaticity as memory provides a comprehensive theoretical approach, which specifies a learning mechanism and predicts the properties of automaticity.

Because automaticity has often been associated with expert (i.e., fast and effortless) performance, most research has focused on its benefits. However, automaticity (or "ritualization," as used in sociolinguistics, and, stronger yet, "mindlessness," as termed by Langer, 1975, in social psychology) has its costs. These costs must be studied for their very important practical as well as their theoretical implications. For that purpose, in the present study we examined the applied problem of verbal checklist procedures and the theoretical approaches to automaticity.

Regardless of the theoretical approach taken, it is generally agreed that "most of the properties of automaticity emerge through practice in consistent environments" (Logan, 1988a, p. 584). The checklist procedure, a fixed list that is always performed in the same manner, constitutes a consistent mapping situation in Schneider and Shiffrin's terminology (Schneider \& Shiffrin, 1977; Shiffrin \& Schneider, 1977). Traditionally, consistent mapping has been considered to be the consistency of the stimulusresponse (S-R) pairing. In the checklist procedure, consistency exists not only in the challenge-response pairing, but also in the presence of the exact same stimuli on every trial and in the fixed sequential order of these stimuli. Thus, crew members have the opportunity to develop automaticity with respect to both item and order information. The typical experimental manipulations used in previous studies have involved only item information. Consequently, consistent and varied mapping have referred to a consistency or variation, respectively, in item information. Schneider and Shiffrin have shown that response automatization and response dissociation occur only under consistent mapping conditions. Varied mapping leads to controlled processing, according to Schneider and Shiffrin, and does not lead to automaticity and dissociation. When order as well as item information is held constant, as in the checklist procedure, a high degree of automaticity can be expected. Because this high degree of automaticity may result from both consistency in item and consistency in order information, it is possible that it could be reduced by variation in order information alone, holding item information constant. 
To provide evidence for the development of automaticity in the checklist procedure and to find ways to interfere with this development, three paper-and-pen experiments were conducted. In the first experiment, subjects timed themselves with stopwatches as they progressed through proofreading material. In the subsequent experiments, the subjects were asked to identify erroneous arithmetic problems embedded in sets of correct problems. In the different experimental conditions, the problems (correct ones as well as erroneous, with the exception of the new set in the alert-new condition of Experiment 3) were identical (i.e., consistent S-R), but the order of operations was manipulated. In addition, following the proofreading task, the subjects were asked to recall the operations used.

In the proofreading task, the subjects were presented with a multiplication problem in which they had to verify the correctness of the product by responding "true" or "false." Similar to the checklist procedure, this task requires four distinct sequential steps. In the checklist procedure, the first step is the call of the item to be checked (e.g., "flaps fifteen degrees"). The second step is the actual action required by the call (e.g., physically placing the flaps lever in the $15^{\circ}$ position), and the third step is the visual verification of the completed action (e.g., checking that the flaps indicator indicates $\left.15^{\circ}\right)$. The fourth and last step in the procedure is the verbal response (e.g., "flaps fifteen degrees").

In the experimental task, these four sequential steps were simulated. The call was simulated by the presentation of a problem (e.g., $2 \times 3=$ ). The second step was a mental multiplication act (e.g., "two times three equals six"). The verification step took place when the subject compared the result of his/her mental multiplication with the printed result on the page (e.g., 6) to verify the printed result, and the response step was simulated by the subject's circling of the appropriate letter (i.e., $T$ for true or $\mathrm{F}$ for false). Furthermore, to simulate the case in which a checklist error is detected and corrected by a flight crew, the subjects were given ballpoint pens. By having them use pens to circle the response letter, it was simple to identify the cases in which subjects first circled the $T$ and then, having realized the error, circled the F.

The second task, recalling the problems presented in the first task, aimed at shedding additional light on the cognitive processes involved in the performance of the first task. Although current theories of automaticity do not make explicit predictions about recall performance, the recall results may shed light on what aspects or components of the task are becoming automatic. In particular, the recall results may provide insight into how the problems are represented by the subjects in the various conditions. For example, because the problems are always presented in a fixed operand order, it will be of interest to determine to what extent subjects' recall responses are consistent with the correct operand order and whether there is a difference between conditions in the extent to which the problems are recalled in the correct operand order. If automaticity is best explained as "single-step direct-access retrieval" (Logan, 1988b, p. 493) from long-term memory, the recall task may allow us to determine what is being retrieved.

\section{EXPERIMENT 1}

Because automaticity is associated with a high speed of performance, a decrease in response times provides evidence for the development of automaticity. Furthermore, automaticity has been shown to lead to powerfunction speedup of performance (Logan, 1988b), so evidence for automaticity is provided to the extent that the decrease in response times follows a power function. Hence, in this initial experiment, we measured the time it took for the subjects to proofread each page of the experimental material. We expected that the proofreading times would decrease as they progressed through the material and that this decrease would follow a power function. Assuming that the consistent sequential order of operations contributes to the development of automaticity beyond the constant $S-R$ pairing (i.e., consistency in order as well as item information is expected to enhance the development of automaticity), we compared a fixedorder condition with a condition in which the sequence of operations was varied (the operations themselves remained constant). We expected that subjects in the fixedorder condition would improve at a faster rate than would those in the varied-order condition, because the varied order would interfere with the development of automaticity.

\section{Method}

Subjects. The subjects were 68 undergraduate psychology students from the University of Colorado, Boulder, with 34 in each condition. In each experimental session, equal numbers of subjects were assigned to each condition, according to a fixed rotation.

Apparatus, Materials, and Design. The apparatus consisted of Advance and Tozaj stopwatches. Each subject was given his or her own watch to use in the experiment.

The material consisted of sets of multiplication problems. Each set included the same 10 problems. The problems were constructed using single-digit operands, not including 1 and 0 . Operands in ascending and descending order were used equally often. Each operation was printed on one line with its solution and the uppercase letters $T$ and $F$. Three sets separated by single lines were printed on a page. Appendix A illustrates a set.

A total of 42 sets was used (i.e., 420 problems on 14 pages). In the fixed-order condition, the 10 multiplication problems were repeated in the same order in all the sets throughout the material. In the varied-order condition, pseudorandom orders were used in such a way that no two sets were in the same order. In both conditions, the multiplication equations were al ways correct; no erroneous problems were included.

Procedure. The subjects were given written instructions, the stopwatches, and the material for the proofreading task and were instructed to proofread the problems. They were also told to circle the $T$ if the product was the correct answer for the given operands, and to circle the $F$ if the product was incorrect. It was explained that accuracy was important, but that the task should be completed as quickly as possible. The subjects were instructed to start the stopwatch when they were given a signal. When they completed each page of the proofreading material, they were to record the time, as indicated on the stopwatch at the bottom of that page. The subjects were told that there was no need for them to stop or restart the stopwatch; as soon as they finished a page, they were to look 
at the time and write down the minutes and seconds in the place provided. They were also told not to waste time on the stopwatch, but to be careful to note the time accurately. Following the completion of the proofreading task, the subjects were instructed to write down as many of the multiplication problems as they could recall. The recall task immediately followed the proofreading task without any delay or intervening tasks.

\section{Results}

Scoring procedure. Proofreading time data were collected from all the subjects. To derive the proofreading time per page, the time indicated at the bottom of each page was subtracted from the time indicated at the bottom of the previous page. In the recall task, problems and operations (operands only, without the product) recalled by the subjects were coded according to their correspondence with the material. This coding included two main categories: problems that were part of the material's standard set and were recalled in the correct order of operands (e.g., $6 \times 3=18$ ) and standard set problems in reverse order of operands (e.g., $3 \times 6=18$ ).

Data analysis. Mean proofreading times were calculated in seconds for each page. In addition, a mixed analysis of variance (ANOVA) was conducted including the between-subjects factor of condition and the withinsubjects factor of page. A second mixed ANOVA was done with the recall results to examine whether there was a significant difference between the correct and reversed orders of operands. In the recall analysis, the withinsubjects factor of order of operands was included along with the between-subjects factor of condition. Although the recall analysis was performed on frequencies, proportions are presented in Table 1.

Reading times. The mean proofreading times for each page of each condition are depicted in Figure 1 on $\log -\log$ coordinates. As is evident, there was a substantial improvement in proofreading times across the pages of the material. The main effect of page was statistically significant $\left[F(13,858)=46.926, M S_{\mathrm{e}}=21.8041, p<.001\right]$. The improvement for the fixed-order condition was more marked than that for the varied-order condition; the interaction of condition and page was significant $[F(13,858)=$ $\left.1.771, M S_{\mathrm{e}}=21.8041, p=.043\right]$.

Because automaticity has been shown to lead to powerfunction speedup, the best-fitting straight lines were fit to the $\log -\log$ functions, as shown in Figure 1. Good fits were obtained for both the fixed-order and the variedorder conditions $\left(r^{2}>.93\right.$ in each case), with a greater slope for the fixed-order $(-0.16096)$ than for the variedorder $(-0.11819)$ condition. These findings suggest that automaticity developed in both conditions, but at a faster rate in the fixed-order condition than in the varied-order condition.

Recall task. The resulting proportions for each group in each of the two categories (standard correct and standard reversed order of operands) as well as the total proportion of standard problems recalled (in either order) are shown in Table 1.

Both groups recalled operations in both the correct and the reversed order of operands. It is interesting to note

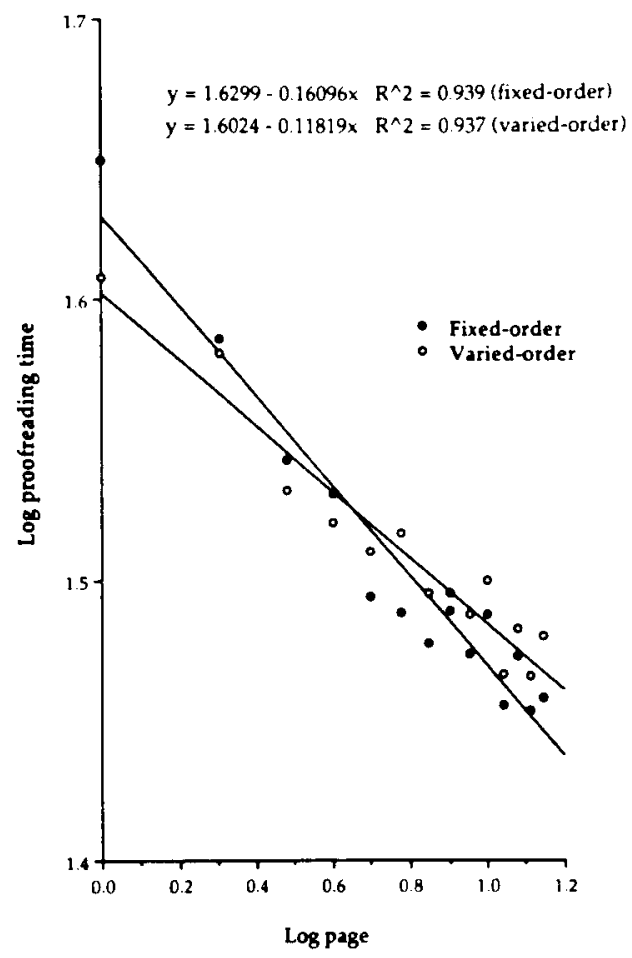

Figure 1. Mean proofreading time (in log seconds) as a function of log page in Experiment 1.

that the fixed-order group recalled more operations in the correct order of operands and fewer operations in the reversed order than did the varied-order group. Even though the main effect of condition was not significant, the interaction of condition and order (correct or reversed) was significant $\left[F(1,66)=5.044, M S_{e}=4.7364, p=\right.$ .026]. Also significant was the main effect of operand order; both groups recalled significantly more operations in the correct order of operands than in the reversed order $\left[F(1,66)=16.470, M S_{\mathrm{e}}=4.7364, p<.001\right]$. The significant interaction suggests that the subjects in the fixedorder condition, in which the problems were in a fixed order, were more sensitive to the displayed order of operands within the problems than were the subjects in the varied-order condition.

\section{EXPERIMENT 2}

In Experiment 1, proofreading speed increased following a power function as the subjects progressed through the experimental material, suggesting that the subjects did indeed develop automaticity. We thus provided evidence that automaticity occurs in this proofreading analog of the checklist procedure and also that sequential order affects the development of automaticity (i.e., there was a smaller decline in proofreading times as a result of varying the order of the operations). To demonstrate the consequences of automaticity on the performance of routine procedures and to provide converging evidence for the effect of sequential order on the development of automatic process- 
Table 1

Proportions for Each Scored Category in the Recall Task of Experiment 1

\begin{tabular}{lcc}
\hline & \multicolumn{2}{c}{ Condition } \\
\cline { 2 - 3 } Scored Category & Fixed-Order & Varied-Order \\
\hline Standard correct & .497 & .441 \\
Standard reversed & .262 & .374 \\
Total & .744 & .812 \\
\hline
\end{tabular}

Note-Standard correct refers to operations from the standard set that were recalled in the correct order of operands; standard reversed refers to operations from the standard set that were recalled in the reversed order of operands. Total refers to operations from the standard set recalled in either the correct or reversed order (when subjects recalled a given operation in both orders, a single entry was made in the total category).

ing, we introduced errors into the proofreading material. We expected the subjects' error-detection ability to reflect the extent to which they had developed automaticity; that is, the more automatic the subjects become, the fewer errors they should be able to detect. Because proofreading times in Experiment 1 leveled off approximately by page 7, the first error was introduced on page 7 of the material in Experiment 2. The training provided by the first six pages was intended to simulate the aviation situation in which pilots have ample opportunities to practice checklist procedures prior to committing any errors. Usually the experienced pilots commit such errors-not the novices, who have not had the opportunity to develop automaticity in this procedure. Also, because errors in aviation checklist procedures are rare, allowing opportunities to reestablish automaticity between occurrences, we included only a few errors in the experimental material.

\section{Method}

Subjects. The subjects were 100 undergraduate psychology students from the University of Colorado, Boulder. Fifty were tested in each of the two conditions. In each experimental session, equal numbers of subjects were assigned to each condition, according to a fixed rotation

Materials and Design. The same sets of multiplication problems that were used in Experiment 1 (see Appendix A) were used. Five of the $\mathbf{4 2}$ sets contained one erroneous problem each, in which one of the operands was replaced with another number. These errors are referred to in the literature as "table-related" (see, e.g., Campbell, 1987) and are known to be difficult to detect. It seemed crucial to make the errors as subtle as possible so that the measurement of proofreading performance would be sensitive and would accurately reflect the type of processing employed by the subjects. The following erroneous problems appeared at various places within the sets: $7 \times 8=63 ; 4 \times 9=27 ; 3 \times 3=6 ; 2 \times 3=8 ; 5 \times 8=10$.

The first error was placed after 20 correct (standard) sets (Problem 204) to allow the subjects to develop a routine of error-free problems. The additional errors were placed in Problems 267, 329, 382 , and 416 , respectively. The sets containing the errors were identical in both conditions and appeared in the exact same places within the material to control for context effects. The different errors occurred in different positions on the page to minimize the chance that the subjects would look for errors in only one place.

In the fixed-order condition, as in Experiment 1, the 10 multiplication problems were repeated in the same order in all the sets throughout the material. In the varied-order condition, pseudorandom orders were used in such a way that no two sets were in the same order, except those sets that contained errors. The sets containing the errors were the only sets repeating the same sequence of operations in the varied-order condition. These sets were identical to the error sets in the fixed-order condition.

Procedure. The subjects were first given written instructions and the material for the proofreading task. Their instructions were the same as those used in Experiment 1, except that there were no instructions concerning the use of a stopwatch. Because changes of response were not prohibited, the subjects were given ballpoint pens to allow the experimenter's identification of such changes. Following the completion of the proofreading task, the subjects were instructed to write down as many of the multiplication problems as they could recall. The recall task followed the proofreading task immediately, without any delay or intervening tasks.

\section{Results}

Scoring procedures. Data were collected and scored for all the subjects. In the proofreading task, the five erroneous problems were coded according to the subjects' marking and the problem's position within the sequence (i.e., 1, 2, 3, 4, or 5). Erroneous problems for which only the $T$ was circled (i.e., the error was not detected) were marked "T." Problems for which both the $\mathrm{T}$ and the $\mathrm{F}$ were circled were marked "T/F," and problems for which only the $F$ was circled were marked " $F$." In addition, the total number of errors detected ( $T / F$ and $F$ combined) was computed. Recall responses were scored as in Experiment 1, but an initial analysis revealed that the existence and detection of erroneous problems contaminated the recall responses (i.e., some but not all of the subjects included some erroneous problems in their recall responses). Hence, we will not report or discuss further the results of the recall task in Experiment 2.

Data analysis. The frequencies for each condition in each of the scored categories were calculated. In addition, ANOVAs were performed on the F, T/F, and total categories to determine whether there was any significant difference between the two conditions. The analysis conducted on the total category (total number of errors detected) included the within-subjects factor of the erroneous problem's position within the sequence. Although the analyses were performed on frequencies, proportions are presented in Table 2 for ease of comparison across experiments.

Proofreading task. The resulting proportions for each group in the $T / F, F$, and total scored categories are shown in Table 2 . As can be seen, both groups in the two conditions marked more erroneous problems as $F$ than as $T / F$, and for each scored category the subjects in the variedorder condition detected more erroneous problems than did those in the fixed-order condition. The difference between the two conditions in the T/F category was not significant $[F(1,98)<1]$. For the $\mathrm{F}$ category, the difference between the two conditions was significant $[F(1,98)=$ 4.725, $\left.M S_{\mathrm{e}}=2.3047, p=.030\right]$. An even larger difference between the two conditions is shown in the total (proportion of errors detected) category $[F(1,98)=$ 8.434, $M S_{\mathrm{e}}=0.2905, p<.005$ ].

The proportions in the total category are shown in Figure 2 as a function of condition and erroneous-problem position in the five-error sequence. Total error detection was significantly influenced by the erroneous problem's position within the error sequence $[F(4,392)=6.630$, 
Table 2

Proportions for the Scored Categories

in the Proofreading Task of Experiment 2

\begin{tabular}{ccc}
\hline & \multicolumn{2}{c}{ Condition } \\
\cline { 2 - 3 } Scored Category & Fixed-Order & Varied-Order \\
\hline T/F & .268 & .276 \\
F & .504 & .636 \\
Total & .772 & .912 \\
\hline
\end{tabular}

Note- $T / F$ represents instances in which subjects circled both $T$ and $F$ responses. $F$ represents instances in which subjects circled only $F$. Total includes both categories.

$\left.M S_{\mathrm{e}}=0.0810, p<.001\right]$. The subjects in the fixed-order condition dramatically improved as they progressed through the material. In contrast, there was no consistent change in the performance of the subjects in the variedorder condition as a function of erroneous-problem position. Thus, the difference between the fixed-order and varied-order conditions was reduced as the subjects progressed through the material; the interaction between condition and erroneous-problem position was significant $\left[F(4,392)=5.247, M S_{\mathrm{e}}=0.0810, p<.001\right]$.

Despite the significant interaction, at every position in the error sequence, the subjects in the varied-order condition outperformed those in the fixed-order condition. The improvement by the fixed-order subjects suggests that detecting an error served to alert them to the possibility of further errors in the experimental material. Perhaps if the errors were more rare or spaced farther apart, as is typical in the aviation checklist procedure, the subjects would have sufficient opportunity to reestablish automaticity between error occurrences. It is crucial to note that the difference between the fixed-order and varied-order conditions is extremely large at the first-error position, which is consistent with the hypothesis that automaticity develops more rapidly in the fixed-order condition than in the varied-order condition. Error detection at the first position is particularly illuminating because it is not contaminated by any side effects of previous error detection; the experimental manipulation of order information is the only factor that could affect the subjects' performance at that point.

\section{EXPERIMENT 3}

The effect of the varied-order condition on the subjects' ability to detect errors in the experimental material and therefore, presumably, on their ability to avoid making errors in performing the checklist procedure, was established in Experiment 2. Varying the order of items in a checklist is, however, not very practical. Many items can only be checked in a specific sequence (e.g., hydraulic pressure can only be checked after the hydraulic pump has been turned on) and changing that sequence would render the whole operation impossible. Nevertheless, it is possible to group together those operations that require a specific sequence, and vary the order of those groups within a given checklist subset (e.g., "Before Starting Engines Checklist"). However, if variation is the key to con- trolled processing, varying the order of items is not the only way to avoid automaticity and thereby increase attention and decrease the probability of error. Variety can be introduced to different aspects of the task (including, of course, varying the stimulus-response mapping, which is the standard manipulation). One type of such variation was the basis for Experiment 3 .

The error-position analysis in Experiment 2 showed that total error detection improved as a function of error position in the fixed-order condition. This finding suggests that detecting errors alerted the subjects to the possibility of further errors in the material. It follows that other variations in the material, not necessarily an erroneous problem, might alert the subjects and thereby improve their detection performance. Accordingly, we introduced two "alerting" manipulations to the fixed-order condition in Experiment 3. In the first condition ("alert-varied"), the set of problems immediately preceding the error set was the set appearing in the same position in the varied-order condition of Experiment 2 (i.e., the set consisted of the same standard problems, but in a different order). In the second condition ("alert-new"), the set of problems immediately preceding the error set was composed of completely new (but correct) problems. The fixed-order condition of Experiment 2 was repeated in order to provide a baseline for assessing performance in these new conditions. Because the largest difference between conditions in Experiment 2 occurred at the first error, and because detecting subsequent errors was contaminated by detection of previous errors, in Experiment 3 the material was terminated two pages after the first (and only) error.

\section{Method}

Subjects. One hundred ninety-five undergraduate psychology students from the University of Colorado, Boulder were tested-65 in each of the three conditions. In each experimental session, equal

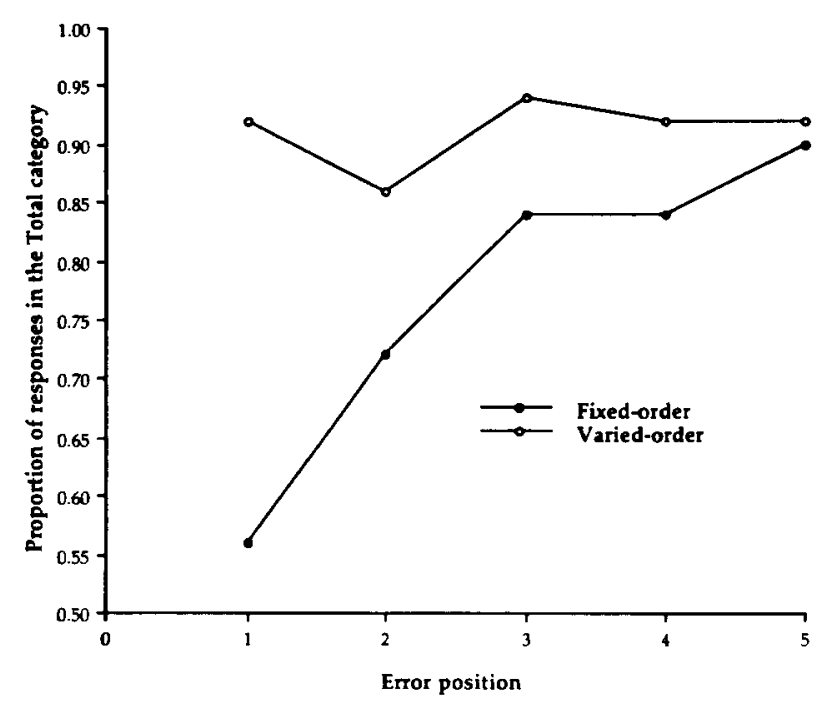

Figure 2. Proportions in the total category as a function of condition and erroneous-problem position in the five-error sequence of Experiment 2. 
numbers of subjects were assigned to each condition, according to a fixed rotation.

Materials and Design. The material for the fixed-order condition was the same as that used for Experiment 2, except that it was terminated two pages after the first error (see Appendix A). Thus, a total of 27 problem sets was used (i.e., 270 problems on nine pages). In the alert-varied condition, the material was the same as for the fixed-order condition in all sets, except the one immediately preceding the set containing the error, which was the same as the parallel set in the varied-order condition of Experiment 2. In the alert-new condition, the material was also the same as for the fixed-order condition in all sets, except the one immediately preceding the set containing the error. That set consisted of entirely new (but correct) problems. Appendix B illustrates the new set.

Procedure. The procedure of Experiment 3 was identical to that of Experiment 2, except that only the first 38 subjects in each condition were given the recall task.

\section{Results}

Scoring procedures. As with Experiment 2, data were collected and scored for all the subjects. The coding and scoring procedures in Experiment 3 were identical to those used in Experiment 2, except that only one error was examined. Recall responses were not scored because of the problem noted in Experiment 2.

Data analysis. The data gathered in Experiment 3 were analyzed as in Experiment 2, allowing for a comparison of the data from the fixed-order, alert-varied, and alertnew conditions. Along with the overall analyses including all three conditions, separate planned analyses were conducted to compare the fixed-order (which was used as a baseline) with each of the other conditions and to compare the two alerting conditions.

Proofreading task. The resulting proportions for each group in the $F, T / F$, and total scored categories are shown in Table 3. Each of the alerting conditions yielded better proofreading performance than did the fixed-order condition in terms of both the F category and the total category (i.e., both by strict and lenient scoring methods). The main effect of condition was significant in the analysis of the F category $\left[F(2,192)=7.278, M S_{\mathrm{e}}=0.2114\right.$, $p=.001]$, and in the analysis of the total category $\left[F(2,192)=4.143, M S_{\mathrm{e}}=0.2240, p=.017\right]$, but not in the analysis of the $\mathrm{T} / \mathrm{F}$ category $[F(2,192)<1]$.

The separate planned analyses revealed significant differences between the fixed-order condition and each of the two alerting conditions in the analysis of the F category [fixed vs. alert-varied, $F(1,128)=4.046, M S_{\mathrm{e}}=$ $0.1901, p=.044$; fixed vs. alert-new, $F(1,128)=$

Table 3

Proportions for the Scored Categories in the Proofreading Task of Experiment 3

\begin{tabular}{cccc} 
& \multicolumn{3}{c}{ Condition } \\
\cline { 2 - 4 } Scored Category & Fixed-Order & Alert-Varied & Alert-New \\
\hline T/F & .323 & .338 & .246 \\
F & .185 & .338 & .492 \\
Total & .508 & .677 & .738 \\
\hline
\end{tabular}

Note-T/F represents instances in which subjects circled both $T$ and $F$ responses. $F$ represents instances in which subjects circled only $F$. Total includes both categories. $\left.15.130, M S_{\mathrm{e}}=0.2034, p<.001\right]$ and in the analysis of the total category [fixed vs. alert-varied, $F(1,128)=$ $3.911, M S_{\mathrm{e}}=0.2380, p=.047$; fixed vs. alert-new, $\left.F(1,128)=7.692, M S_{\mathrm{e}}=0.2250, p=.006\right]$, but not in the analysis of the $T / F$ category [fixed vs. alert-varied, $F(1,128)<1$; fixed vs. alert-new, $F(1,128)<1$ ].

There was, however, no significant difference between the two alerting conditions according to the analyses of the total category $[F(1,128)<1]$ and the $T / F$ category $\left[F(1,128)=1.332, M S_{e}=0.2079, p=.249\right]$. The analysis of the $F$ category revealed a marginally significant advantage for the alert-new condition $[F(1,128)=3.197$, $\left.M S_{\mathrm{e}}=0.2406, p=.072\right]$. It is interesting to note that the three conditions showed different patterns of errordetection responses. The proportion of total error detection in the F category (i.e., those detected immediately) was highest in the alert-new condition, whereas the proportion of total error detection in the T/F category (i.e., those detected only after an initial incorrect response) was lowest in the alert-new condition. This pattern of conditional error proportions is illustrated in Figure 3.

\section{GENERAL DISCUSSION}

The response times in Experiment 1 provide evidence that the subjects developed automaticity as they progressed through the proofreading material. When errors were introduced into the material in Experiment 2, the results of the proofreading task show that the subjects in the variedorder condition detected significantly more errors than did those in the fixed-order condition. The error-detection results suggest that the subjects in the fixed-order condition paid less attention to the task than did those in the varied-order condition. This finding is consistent with the hypothesis that a fixed-order condition produces more automaticity than does a varied-order condition. Both theories of automaticity, automaticity as resource allocation and automaticity as memory, would predict these results if the constant sequential order of operations is accepted as a consistent environment. What these results show is that, indeed, a constant sequential order of operations should be considered as a consistent environment that augments constant $\mathrm{S}-\mathrm{R}$ mapping and, hence, promotes automatic processing. Because item information was held constant, even in the varied-order condition, it is likely that the subjects in both conditions developed automaticity to some extent. However, the significant effect of condition on response times in Experiment 1 and on error detection in Experiment 2, and the large difference between the fixed-order and the varied-order conditions in the detection of the first error in Experiment 2 suggest that varying order information has a crucial impact on the development of automaticity.

Anecdotal experience (e.g., a near accident seems to alert all involved to the possibility of a future actual accident) suggests that it may be possible to affect subjects' habitual response to a familiar pattern by introducing an alerting stimulus. Indeed, both the error-position analysis of Experiment 2 and the results of the proofreading 


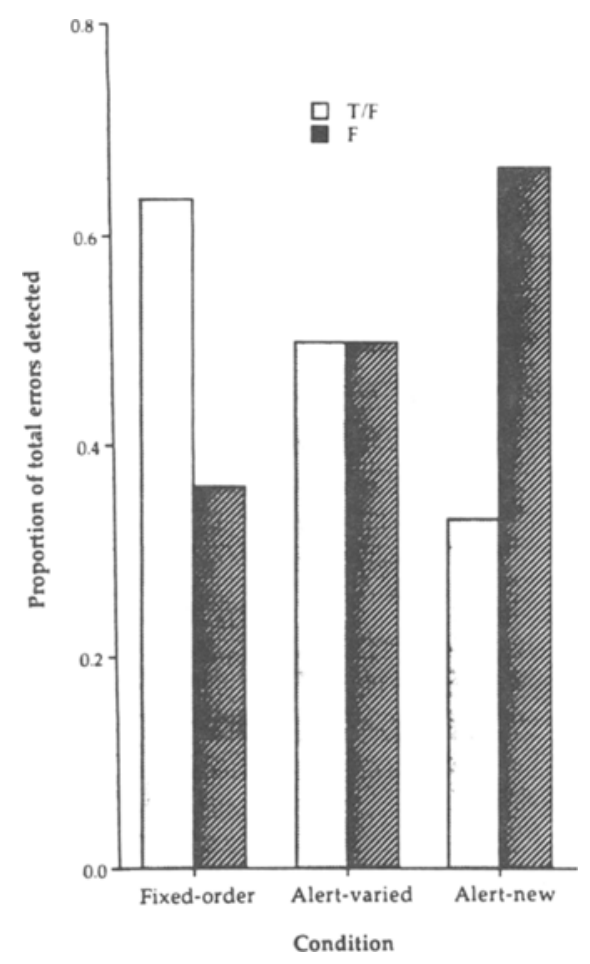

Figure 3. Proportions of total error-detection responses in the $\mathbf{F}$ and $T / F$ categories as a function of condition.

task in Experiment 3 show that introducing alerting stimuli significantly changes subjects' error-detection performance. This significant change could be explained in one of two ways. The alert could be seen as directly interfering with the automatic processing of the material. However, it has been claimed that it is very difficult to interfere with automatic responding once automaticity has been established (see, e.g., Shiffrin \& Schneider, 1977). To avoid such an objection, it could be argued, alternatively, that the alert could lead to an increased tendency to perform a rechecking process (see, e.g., Krueger, 1978, for a discussion of rechecking processes). The $\mathrm{T} / \mathrm{F}$ response (in which subjects circle the $T$, usually cross it out, and also circle the $F$ ) suggests that subjects indeed employ a second, and probably slower, controlled process that involves rechecking. Such rechecking is evident to some extent even in the fixed-order condition.

The present data do not allow us to discriminate between these two possible explanations for the significant effects of the alerting conditions in Experiment 3. The significant difference between the alerting conditions and the fixed-order condition in the F and total categories and the lack of a significant difference in the $T / F$ category suggest that the alerting stimuli directly interfered with the automatic responses. However, it is also possible that a rechecking process may lead to an $F$ rather than a $T / F$ response if subjects initially make an implicit automatic $T$ response, but then inhibit the explicit circling of the $T$ and, following the recheck, circle only the F. Hence, the observed pattern of results is consistent with the hy- pothesis that alerting stimuli increase rechecking if it is assumed that they also enhance the inhibition of the initial implicit automatic responses.

The results of the recall task of Experiment 1 bring up several interesting theoretical issues. At present, theories of automaticity do not make any explicit statements about the relationship between controlled versus automatic processing and memory strength. It is possible that the total number of operations recalled under each experimental condition is the result of several different forces (e.g., encoding variability; see Melton, 1970) that are independent of the underlying automatic or controlled processing. Nevertheless, because automaticity was greater in the fixed-order condition than in the varied-order condition (according to the differences in the proofreading times), examining the differences between the conditions in the specific operations that are recalled illuminates the underlying automatic processes and representations.

Because the problems in both conditions always appeared in the same operand order, we examined the extent to which subjects recalled the operations in the correct or reversed order of operands. The fact that the subjects in the fixed-order condition recalled more operations in the correct order and fewer in the reversed order than did those in the varied-order condition suggests that the subjects in the fixed-order condition were more sensitive to the displayed order of operands. Under Logan's (1988b) proposal that automatic responding consists of a "singlestep direct-access retrieval" (p. 493) from long-term memory, this finding implies that the relevant memory representation is order-specific. In other words, the results suggest that what seems to be automatic in our task is the association of the whole problem as an ordered pattern with the $T$ response. For example, the subjects associated the pattern " $3 \times 6=18$," but not " $6 \times 3=$ 18 ," with the $T$ response.

Furthermore, the present recall data are important to another related issue concerning memory representations. The mere fact that operations were frequently recalled in the reversed order of operands is of interest. Fendrich, Healy, and Bourne (1993) address the issue of the representation of commuted pairs: "problems that differ only in operand order (e.g., $3 \times 4$ and $4 \times 3$ )" ' (p. 114). They note that commuted pairs "are of concern to models of mental arithmetic because at present it is unclear whether they should be represented as two different problems, as two instances of the same problem, or as a hierarchy of two problems attached to a common node" (p. 114). In their experiments, they found evidence for the unique representation "with an overlap, possibly via a higher order node, in the mental representation of the commuted problems" (p. 131). Fendrich et al. point out that "this interpretation based on independent or distinct representations cannot be distinguished on the basis of the current data alone from an alternative interpretation, which asserts a single representation for commuted operands with different retrieval or access routes depending on operand order... Nonetheless," they maintain, "the present findings suggest that models of mental multiplication should 
not assume complete independence of commuted pairs of problems"' (p. 131). The results presented here are consistent with these findings.

The present evidence for the above suggestion comes from two observations - that subjects recalled operations in both correct and reversed orders of operands, and that they recalled significantly more operations in the correct order than in the reversed order of operands. If commuted pairs are stored together with no distinctions, their recall should show an equal distribution. The significant difference in distribution speaks against such common storage or lack of distinctions. The mere recall of reversed-order operations, however, is particularly interesting in light of an important difference between the two studies.

Fendrich et al. (1993) tested subjects for recognition with operations in both orders of operands (commuted pairs). The present study presented subjects with operations in one order of operands only, and tested subjects for recall. The fact that the subjects in the present study reported (recalled?) a large number of operations in the reversed (erroneous) order of operands, that they sometimes reported the same operations in both orders, and that the first operation that they reported (assuming that the order in which the operations were written represents the order of recall) was often in the reversed order, presents a problem not only to models of mental multiplication, but also to models of memory. The two main questions these models would have to answer to account for the data in the present study are: (1) why did the subjects remember the operations as being in both orders of operands? and (2) once written down, why were the reversed operand order operations not recognized as being "incorrect?' Again, the simple response that a single representation of commuted pairs answers these questions would not suffice as the distribution issue discussed above indicates.

As for the checklist procedure, it presents a paradoxical situation with respect to automaticity. As mentioned earlier, automaticity is often associated with expert performance. The ability to perform tasks quickly and effortlessly while attending to another task is the mark of the expert. Pilots must be experts; they must be able to perform their routine tasks (such as flying the aircraft, monitoring instruments, and following checklists) quickly and effortlessly, while continuously watching for potential problems. However, because the routine tasks are so complex and susceptible to errors, they must be executed under controlled processing to avoid such errors. Thus, pilots are asked to do the impossible; they are asked to develop automatic-like performance while maintaining controlled processing. One way to make their job more feasible is to introduce some variation into the checklist procedure.

Variation can be incorporated into the checklist procedure in one of three ways (or a combination thereof): by varying the challenge (stimulus) or the response, by varying the order of operations, or by introducing alerting stimuli, as we have shown in Experiment 3. The significant difference between the alerting conditions and the fixed-order condition of Experiment 3 implies that incorporating an alerting stimulus into a checklist reduces the likelihood of errors in the procedure. Even though there were no significant differences between the two alerting conditions of Experiment 3, the marginally significant advantage for the alert-new condition in the F category and the difference in the pattern of detection responses suggests that the alert-new condition may be preferred in a practical setting where an initial $T$ response, even if followed by a subsequent $F$ response, could lead to a disaster. As illustrated in Figure 3 and in Table 3, in the alert-new condition there were twice as many detection responses in the $F$ category as in the $T / F$ category, whereas in the alert-varied condition the detection responses were evenly split between the $F$ and $T / F$ categories. We intend to conduct additional studies to determine which type of variation is most effective in preventing automaticity while minimizing changes to the checklist itself. Ultimately, studies with flight crews are required to find the most practical application of automaticity theory to the checklist procedure in the cockpit. The importance of optimizing the checklist procedure in the cockpit is underscored by the conclusion of a recent report describing a fatal crash: "The air carrier crew at LGA [New York La Guardia Airport] went through the motions of reading a checklist but responded without being effective"' (Olcott, 1991).

\section{REFERENCES}

BARGH, J. A. (1984). Automatic and conscious processing of social information. In R. S. Wyer, Jr., \& T. K. Srull (Eds.), Handbook of social cognition (Vol. 3, pp. 1-37). Hillsdale, NJ: Erlbaum.

Campbell, J. I. D. (1987). Production, verification, and priming of multiplication facts. Memory \& Cognition, 15, 349-364.

Cheng, P. W. (1985). Restructuring versus automaticity: Alternative accounts of skill acquisition. Psychological Review, 92, 414-423.

Clark, H. H. (1979). Responding to indirect speech acts. Cognitive Psychology, 11, 430-477.

DEGANI, A., \& WIENER, E. L. (1990). Human factors of flight-deck checklists: The normal checklist (Report No. 177549). Moffett Field, CA: NASA-Ames Research Center.

DUNCAN, J. (1986). Consistent and varied training in the theory of automatic and controlled processing. Cognition, 23, 279-284.

Fendrich, D. W., Healy, A. F., \&ourne, L. E., Jr. (1993). Mental arithmetic: Training and retention of multiplication skill. In C. Izawa (Ed.), Cognitive psychology applied (pp. 111-133). Hillsdale, NJ: Erlbaum.

Fisk, A. D., Ackerman, P. L., \& Schneider, W. (1987). Automatic and controlled processing theory and its application to human factors problems. In P. A. Hancock (Ed.), Human factors psychology (pp. 159-197). New York: North-Holland.

Fisk, A. D., Eвосн, M. (1989). An automatic/controlled processing theory application to training component map reading skills. $A p$ plied Ergonomics, 20, 2-8.

Fisk, A. D., \& GAlliNı, J. K. (1989). Training consistent components of task: Developing an instructional system based on automatic/controlled processing principles. Human Factors, 31, 453-463.

Gumperz, J. J., \& Hymes, D. (EDS.). (1972). Directions in sociolinguistics. New York: Holt, Rinehan \& Winston.

HASHER, L.. \& ZaCkS, R. T. (1979). Automatic and effortful processes in memory. Journal of Experimental Psychology: General, 108, 356-388.

Hawkins, F. H. (1987). Human factors in fight. Brookfield, VT: Gower. 
Klapp, S. T., Boches, C. A., Trabert, M. L., \& Logan, G. D. (1991). Automatizing alphabet arithmetic: 2 . Are there practice effects after automaticity? Journal of Experimental Psychology: Leaming, Memory, \& Cognition, 17, 194-207.

Krueger, L. E. (1978). A theory of perceptual matching. Psychological Review, 85, 278-304.

LaBerge, D., \& Samuels, S. J. (1974). Toward a theory of automatic information processing in reading. Cognitive Psychology, 6, 293-323.

LANGER, E. J. (1975). The illusion of control. Journal of Personality \& Social Psychology, 32, 311-328.

LANGer, E. J. (1989). Mindfulness, Reading, MA: Addison-Wesley.

LOGAN, G. D. (1978). Attention in character classification: Evidence for the automaticity of component stages. Journal of Experimental Psychology: General, 107, 32-63.

LoGAN, G. D. (1988a). Automaticity, resources, and memory: Theoretical control ersies and practical implications. Human Factors, 30 , 583-598.

LOGAN, G. D. (19881). Toward an instance theory of automatization. Psychological Review, 95, 492-527.

LoGAN, G. D., \& KLAPP, S. T. (1991). Automatizing alphabet arithmetic: 1 . Is extended practice necessary to produce automaticity? Journal of Experimental Psychology: Learning, Memory, \& Cognition, 17, 179-193.

MELTON, A. W. (1970). The situation with respect to the spacing of repetitions and memory. Journal of Verbal Learning \& Verbal Behavior, 9, 596-606.

Newell, A., \& Rosenbloom, P. S. (1981). Mechanisms of skill acquisition and the law of practice. In J. R. Anderson (Ed.), Cognitive skills and their acquisition (pp. 1-55). Hillsdale, NJ: Erlbaum.

OucotT, J. W. (1991, October). Check complacency. Business \& Commercial Aviation, 67, p. 134.

Posner, M. I., \& SNyder, C. R. R. (1975). Attention and cognitive control. In R. L. Solso (Ed.), Information processing and cognition: The Loyola symposium (pp. 55-85). Hillsdale, NJ: Erlbaum.

Rosenbloom, P. S., \& NeWEll, A. (1986). The chunking of goal hierarchies: A generalized model of practice. In R. S. Michalski, J. G. Carbonell, \& T. M. Mitchell (Eds.), Machine learning: An artificial intelligence approach (Vol. 2, pp. 247-288). Los Altos, CA: Morgan Kaufmann.

RYAN, C. (1983). Reassessing the automaticity-control distinction: Item recognition as a paradigm case. Psychological Review, 90, 171-178.

SCHIEFFELIN, B. B., \& OCHS, E. (EDS.). (1986). Language socialization across cultures. Cambridge: Cambridge University Press.

SCHNEIDER, W. (1985). Toward a model of attention and the development of automatic processing. In M. I. Posner \& O. S. Marin (Eds.), Attention and performance XI (pp. 475-492). Hillsdale, NJ: Erlbaum.

SCHNEIDER, W., \& DeTweiler, M. (1987). A connectionist/control architecture for working memory. In G. H. Bower (Ed.), The psychology of learning and motivation (Vol. 21, pp. 53-119). San Diego, CA: Academic Press.

SChneider, W., Dumais, S. T., \& Shiffrun, R. M. (1984). Automatic and control processing and attention. In R. Parasuraman \& R. Davies (Eds.), Varieties of attention (pp. 1-27). San Diego, CA: Academic Press.

SCHNEIDER, W., \& ShIFfrin, R. M. (1977). Controlled and automatic human information processing: 1. Detection, search and attention Psychological Review, 84, 1-66.

Shiffrin, R. M., \& SCHNeIDer, W. (1977). Controlled and automatic human information processing: 2. Perceptual learning, automatic attending and a general theory. Psychological Review, 84, 127-129.

Swain, A. D., \& GutTman, H. E. (1983). Handbook of human reliability analysis with emphasis on nuclear power plant applications (NUREG/CR-1278, SAND80-00200). Washington, DC: U.S. Nuclear Regulatory Commission.

\section{APPENDIX A}

Sample Standard Set of Multiplication Problems

$\begin{array}{llll}6 \times 3=18 & \text { T } & \text { F } \\ 2 \times 4=8 & \text { T } & \text { F } \\ 8 \times 5=40 & \text { T } & \text { F } \\ 7 \times 9=63 & \text { T } & \text { F } \\ 4 \times 7=28 & \text { T } & \text { F } \\ 5 \times 2=10 & \text { T } & \text { F } \\ 3 \times 9=27 & \text { T } & \text { F } \\ 8 \times 6=48 & \text { T } & \text { F } \\ 3 \times 2=6 & \text { T } & \text { F } \\ 4 \times 8=32 & \text { T } & \text { F }\end{array}$

\section{APPENDIX B}

The Alert-New Set of Multiplication Problems in Experiment 3

$\begin{array}{lll}5 \times 4=20 & \text { T } & \text { F } \\ 2 \times 6=12 & \text { T } & \text { F } \\ 9 \times 4=36 & \text { T } & \text { F } \\ 2 \times 7=14 & \text { T } & \text { F } \\ 3 \times 3=9 & \text { T } & \text { F } \\ 7 \times 5=35 & \text { T } & \text { F } \\ 3 \times 8=24 & \text { T } & \text { F } \\ 2 \times 9=18 & \text { T } & \text { F } \\ 8 \times 2=16 & \text { T } & \text { F } \\ 5 \times 5=25 & \text { T } & \text { F }\end{array}$

(Manuscript received June 27, 1991; revision accepted for publication December 30, 1992.) 